\title{
Reproductive outcomes in women and men using complementary and alternative medicine treatment and not receiving artificial reproductive technology: a systematic review
}

\author{
Hannah M. Yogasundram ${ }^{1}$ - Andrew J. O. Hui ${ }^{1}$ - Clifford Y. S. Sia ${ }^{1}$. Anthea C. Chui ${ }^{1}$ - William J. Waldock ${ }^{1}$. \\ Siobhan Quenby ${ }^{2,3}$ (D) Elizabeth Brown ${ }^{4}$. Clare Oliver-Williams ${ }^{5,6}$
}

Received: 4 June 2020 / Accepted: 7 October 2020 / Published online: 20 October 2020

(c) The Author(s) 2020, corrected publication 2020

\begin{abstract}
Purpose Infertility is a global problem, but only a minority of couples access assisted reproductive technologies due to financial and sociocultural barriers. Complementary and alternative medicine are seen as another option. We aimed to determine the impact of complementary and alternative medicine on conception, miscarriage and live birth rates in couples not receiving assisted reproductive technology treatments.

Methods The electronic databases EMBASE, PubMed, Web of Science and the Allied and Complementary Medicine Database were systematically searched before March 24th 2020. Reference lists of eligible studies were searched for relevant studies. Eligible studies included trials and observational studies that assessed a complementary or alternative medicine and conception, miscarriage or live births in men or women not undergoing fertility treatment. Data were extracted by two independent reviewers using a pre-designed data collection form. The study protocol was published in the PROSPERO database (CRD42018086980).

Results Twenty randomized controlled trials were identified, including 2748 individuals. Most studies did not demonstrate any effect of a complementary or alternative medicine on pregnancy, live birth or miscarriage rates. Limited evidence was found for a positive effect of herbal therapies taken by women on conception rates. There was substantial diversity in quality across the studies.

Conclusion There is limited evidence of the effectiveness of complementary and alternative medicine on improving the chances of conception and live births, or increasing miscarriage risk. Owing to the generally sub-optimal quality and heterogeneous nature of the evidence, rigorous studies are needed to determine the impact of complementary and alternative medicine on fertility.
\end{abstract}

Keywords Pregnancy $\cdot$ Conception $\cdot$ Live births $\cdot$ Miscarriage $\cdot$ Complementary medicine $\cdot$ Alternative medicine

Hannah M. Yogasundram and Andrew J. O. Hui are lead authors.

Electronic supplementary material The online version of this article (https://doi.org/10.1007/s00404-020-05836-4) contains supplementary material, which is available to authorized users.

Clare Oliver-Williams

cto21@cam.ac.uk

1 School of Clinical Medicine, University of Cambridge, Cambridge, UK

2 Warwick Medical School, University of Warwick, Coventry, UK

3 University Hospitals Coventry and Warwickshire, Coventry, UK

\section{Abbreviations}

ART Assisted reproductive technology treatment CAM Complementary or alternative medicine PCOS Polycystic ovary syndrome

4 University College London, London, UK

5 Homerton College, University of Cambridge, Hills Road, Cambridge, UK

6 Department of Public Health and Primary Care, University of Cambridge, 2 Worts' Causeway, Cambridge CB1 8RN, UK 


\section{Introduction}

Infertility is a significant problem which affects $7-16 \%$ of couples seeking to have children [1, 2]. Assisted reproductive technology (ART) treatment is regularly used to help couples experiencing infertility to have a child. However, inequity in access to ART treatment have been well reported, [3-5] and there is evidence for a declining trend in the use of ART treatment in the US [6].

Given the disproportionate access to ART treatment as well as its potentially high cost, alternatives are often sought by couples. Use of complementary and alternative medicines (CAM) to treat infertility is common. This may be because people perceive CAM to be of lower cost, safer or more effective [7]. A 2010 prevalence study from eight American community and academic infertility practices found that $29 \%$ of couples had used a CAM for infertility, with $22 \%$ trying acupuncture [8].

There is a broad range of CAM that purport to have a positive effect on fertility including traditional practices such as acupuncture: Chinese medicine remedies; use of naturopaths, homeopaths and chiropractors; and cognitive and mind-body therapies, such as mindfulness. Although the potential impact of these treatments on infertility has been assessed within the context of ART treatment [9], this has limited interpretation for couples who are not receiving ART treatment.

Considering the increasingly wide-ranging use of CAM by couples trying to conceive and the inequity in access to ART treatment, a comprehensive review on the efficacy of CAM treatments in couples not receiving ART treatment is essential. Therefore, we present a systematic review of all available intervention and observational studies to review the impact of a range of CAM on conception, miscarriage or live births rates in couples not receiving ART treatment.

\section{Methods}

\section{Information sources and search strategy}

This review was conducted using a predefined protocol, in accordance with PRISMA guidelines (eTable 1) and is registered on PROSPERO [10] (CRD42018086980). Four electronic databases (EMBASE, PubMed, Web of Science and the Allied and Complementary Medicine Database) were searched until 24th March 2020 without time and language restriction. The computer-based searches combined terms related to (1) the interventions, such as alternative or complimentary natural therapies (including herbal therapies, traditional Chinese medicine, ayurveda, naturopathy, chiropractic, osteopathy, massage, yoga, relaxation therapy, homeopathy, aromatherapy and therapeutic touch) (2) pregnancy and related outcomes (e.g., conception, miscarriage, birth) and (3) study design (e.g. RCT, longitudinal study) in humans (eTable 2). CAM were identified through previous CAM publications [11-13] to aid comparison and popular fertility websites which provide advice to couples (https://www.fertilityiq.com; https://www.babycentre .co.uk; https://www.oviahealth.com). Two independent reviewers (EB, WW, AC, HY, CS, AH) screened the titles and abstracts of all studies initially identified according to the selection criteria, and any disagreement was resolved through consensus or consultation with a third independent reviewer (CO). Full texts were retrieved from studies that satisfied all selection criteria. Reference lists of studies and reviews identified on the topic were searched to identify additional publications.

\section{Selection criteria}

Studies were eligible if they: (1) were longitudinal studies, randomized controlled trials (RCTs) or non-randomized trials; (2) assessed effects of any CAM (list in eTable 3 ) in men or women seeking to conceive; (3) collected relevant endpoints, including conception, miscarriage or live births rates; and (4) control and treatments groups only differed on the administration of a CAM. Study populations included women or men experiencing sub-fertility or infertility due to a diagnosed medical condition (e.g. polycystic ovary syndrome (PCOS), diminished ovarian reserve) or due to an unexplained or unspecified condition. The participants could not be receiving in vitro fertilization (IVF) or another ART treatment at the time of the trial. No restrictions on length of follow-up or language were applied.

\section{Data extraction}

The exposures or interventions eligible for inclusion in the current review were summarized using the following broad groupings: (i) biologically based therapies such as herbal extracts and dietary supplements not conventionally recommended for conception or pregnancy (i.e. folic acid would not be eligible); (ii) mind-body and behavioral therapies (e.g. mindfulness); (iii) body manipulation and energy therapies (e.g. acupuncture); and (iii) traditional (alternative) medical systems (e.g. homeopathy). Two authors independently extracted data (EB, WW, AC, HY, CS, AH) and a consensus was reached in case of any inconsistency with involvement of a third author (CO). A predesigned electronic data abstraction form was used to extract relevant information. The form included the following: author, year, study design, country, study years, number of participants, number lost to follow-up, recruitment process 
and inclusion/exclusion criteria, CAM, category of CAM (Biological/Energy/Body manipulation/Traditional medical systems), control group intervention, outcome(s), the number receiving the CAM, follow-up duration, average age of participants, pregnancies in treatment/control groups ( $N$ or $\%)$, miscarriages in treatment/control groups $(N$ or $\%)$, live births in treatment/control groups ( $N$ or \%), risk estimate for the difference between the therapy and control group, adverse events in the CAM group and an open text field for additional notes. In case of multiple publications on a study, the most up-to-date or comprehensive information was extracted.

\section{Statistical analysis}

For studies which did not statistically compare the rates of the outcome by exposure group the 2 -sided chi-squared test was conducted using the raw data provided in the manuscript. Analyses were conducted Stata version 12.0 (Stata Corp, College Station, Texas, USA). All P values from 2 -sided tests were considered statistically significant at $p<0.05$.

\section{Assessing the risk of bias}

Two reviewers independently rated the quality of studies using the Cochrane Collaboration's tool (HMY, CO) [14]. This tool evaluated seven domains for their risk of bias (high, low, or unclear) as follows: random sequence generation, allocation concealment, blinding of participants and personnel, blinding of outcome assessment, incomplete outcome data, selective reporting and other biases. We provided an overall assessment of the risk of bias in each study as follows: if a study had no domains considered at high risk and at least four domains deemed low risk (at least one of them being random sequence generation or allocation concealment), the study was deemed low risk; if a study had one domain at high risk it was deemed to be a high risk study; otherwise it was deemed to be of unclear risk. Any disagreements were discussed until a consensus was reached.

To assess the overall quality of evidence, two authors applied the Grading of Recommendations Assessment, Development and Evaluation (GRADE) approach. As individual studies were assessed using the Cochrane Collaboration's tool, assessment of the GRADE approach was only applied where two or more studies were identified for an exposure-outcome combination. We downgraded the evidence from high quality by one level for each serious study limitation that was identified. Study limitations were the following: risk of bias, indirectness of evidence, serious inconsistency, imprecision of effect estimates or potential publication bias. Two or more studies were identified for the following exposure and outcome combinations and were, therefore, included in the GRADE assessment: antioxidants for men and conception; herbal therapies for women and conception, acupuncture for women and conception; and homeopathy for women and live births.

Meta-analyses were not conducted in part because the majority of studies did not assess the same exposure and outcome. The few studies that did evaluate the same exposure-outcome combination had important methodological heterogeneity, including study quality, population studied and study design, which was reflected in statistical heterogeneity in a preliminary meta-analysis.

\section{Results}

We identified 27,085 publications in our search strategy after removal of duplicates. After assessment, 20 original articles were identified that evaluated conception, miscarriage or live births after the use a CAM (Fig. 1) [15-34]. Table 1, Fig. 2 and eTable 4 summarize the studies included in the review. The results of these studies are presented by endpoint in order of the direction of evidence. The quality of evidence from these studies is summarized in Table 2. The interventions and results of the studies evaluating conception, live births and miscarriages are summarized in eTables 5-7, respectively. The included studies evaluated a biological CAM $(n=13)$, energy therapies ( $n=4)$, body manipulations $(n=1)$, traditional CAM $(n=1)$ or a combination $(n=1)$.

\section{Conception}

Twenty studies investigated conception after treatment with a CAM (eTable 5). All studies were RCT. Eleven studies were carried out in Europe, three in the USA, two in Australia, one each in China, Iran, India and one in China and Australia together. Women and men were treated in ten studies each. Thirteen studies assessed biological based therapies, four studied energy therapies, one each studied body manipulation and traditional methods and one assessed a combination of body manipulation practices and traditional CAM. Of the biological therapies, there were ten antioxidants, one herbal supplement, one dietary supplement and one combined herbal and dietary supplement.

Seven studies found evidence for a positive effect of CAM treatment on conception and 12 did not show a difference.

\section{Studies reporting no statistically significant effect}

Six studies found no statistically significant effect of antioxidants in sexually active men experiencing infertility. Balercia et al. [15] studied the effect of co-enzyme Q10 (200 mg/ day) taken for 6 months with 3 months' follow-up in a placebo-controlled, double-blind RCT of 60 sexually active 


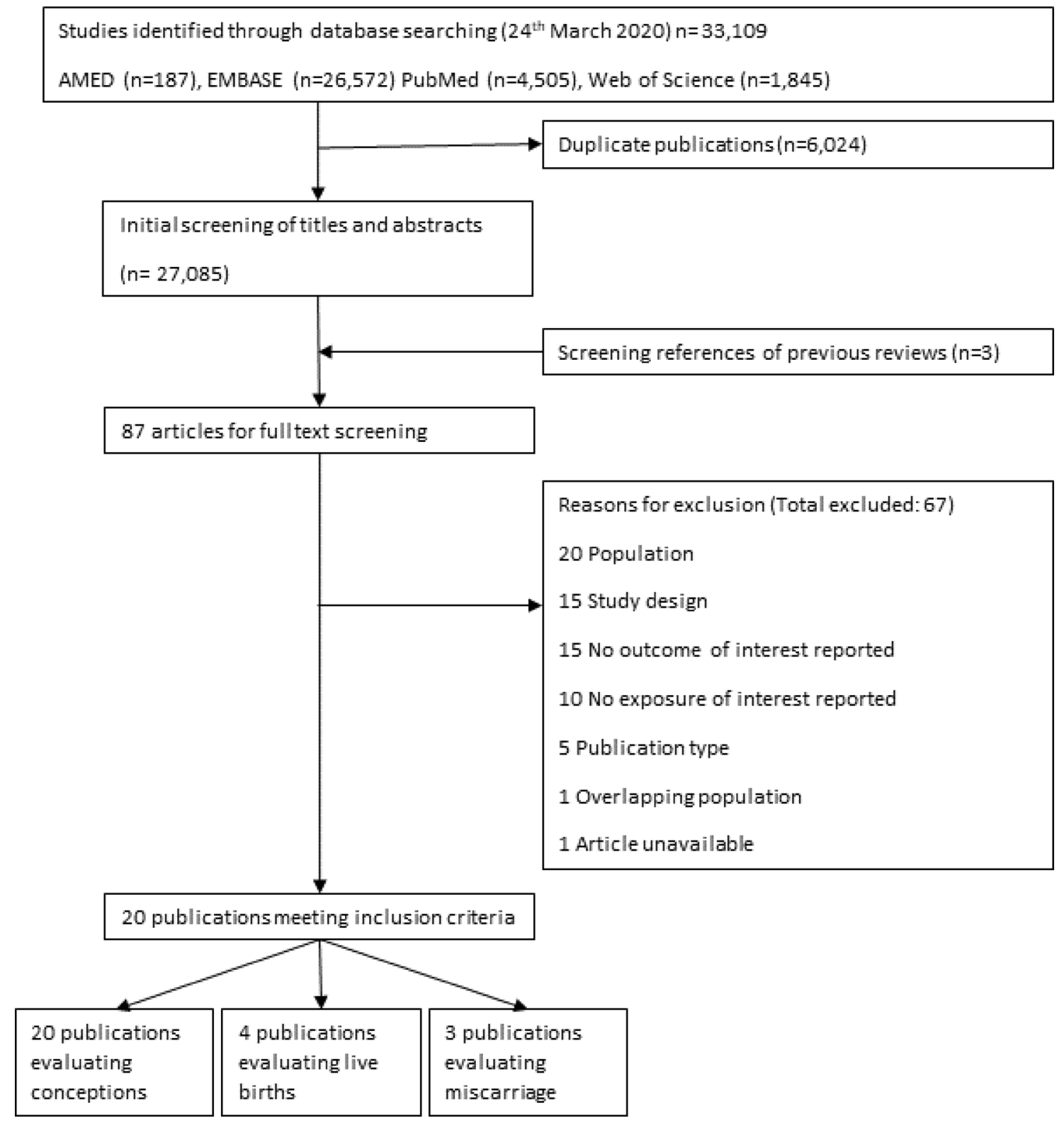

Fig. 1 Flow diagram of identification and selection of studies included in the systematic review

men with idiopathic infertility. In the treatment group, six partners became pregnant compared to three in the control group $(20.0 \%$ vs $13.0 \% p=0.301)$.

In a factorial, placebo-controlled, double-blind RCT, Balercia et al. [17] assessed the effect of L-carnitine ( $3 \mathrm{~g} /$ day), L-acetyl-carnitine (3 g/day), or combined L-carnitine ( $2 \mathrm{~g}$ /day) and L-acetyl-carnitine $(1 \mathrm{~g} /$ day $)$ over a 6 -month period with 3 months' follow-up on 60 men with idiopathic infertility. Two partners became pregnant in each of the single therapy groups, compared to three in the control group $(13.3 \%$ and $13.3 \%$ vs $20.0 \%, p=0.624)$. Five partners became pregnant in the combined therapy group $(33.3 \%$ vs $20.0 \%, p=0.409)$.
Gopinath et al. [16] evaluated the effect of antioxidants on 138 men with subfertility in a three-arm, double-blind, placebo-controlled RCT. Men received either two tablets of antioxidants a day (50 mg co-enzyme Q10, $500 \mathrm{mg}$ L-carnitine, $2.5 \mathrm{~g}$ lycopene, $12.5 \mathrm{mg}$ zinc), one tablet of antioxidants and one placebo tablet, or two placebo tablets for 180 days, with up to 6 months of follow-up. There were six conceptions for men receiving two tablets of antioxidants, seven for men receiving one tablet of antioxidant and two in the control group, $(13.0 \%$ vs $5.6 \%, p=0.257)$ and $(16.3 \%$ vs $5.6 \%, p=0.135)$, respectively.

Sigman et al. [18] evaluated the effect of 24 weeks of L-carnitine (2 g/day) and L-acetyl-carnitine ( $1 \mathrm{~g} /$ day $)$ on 


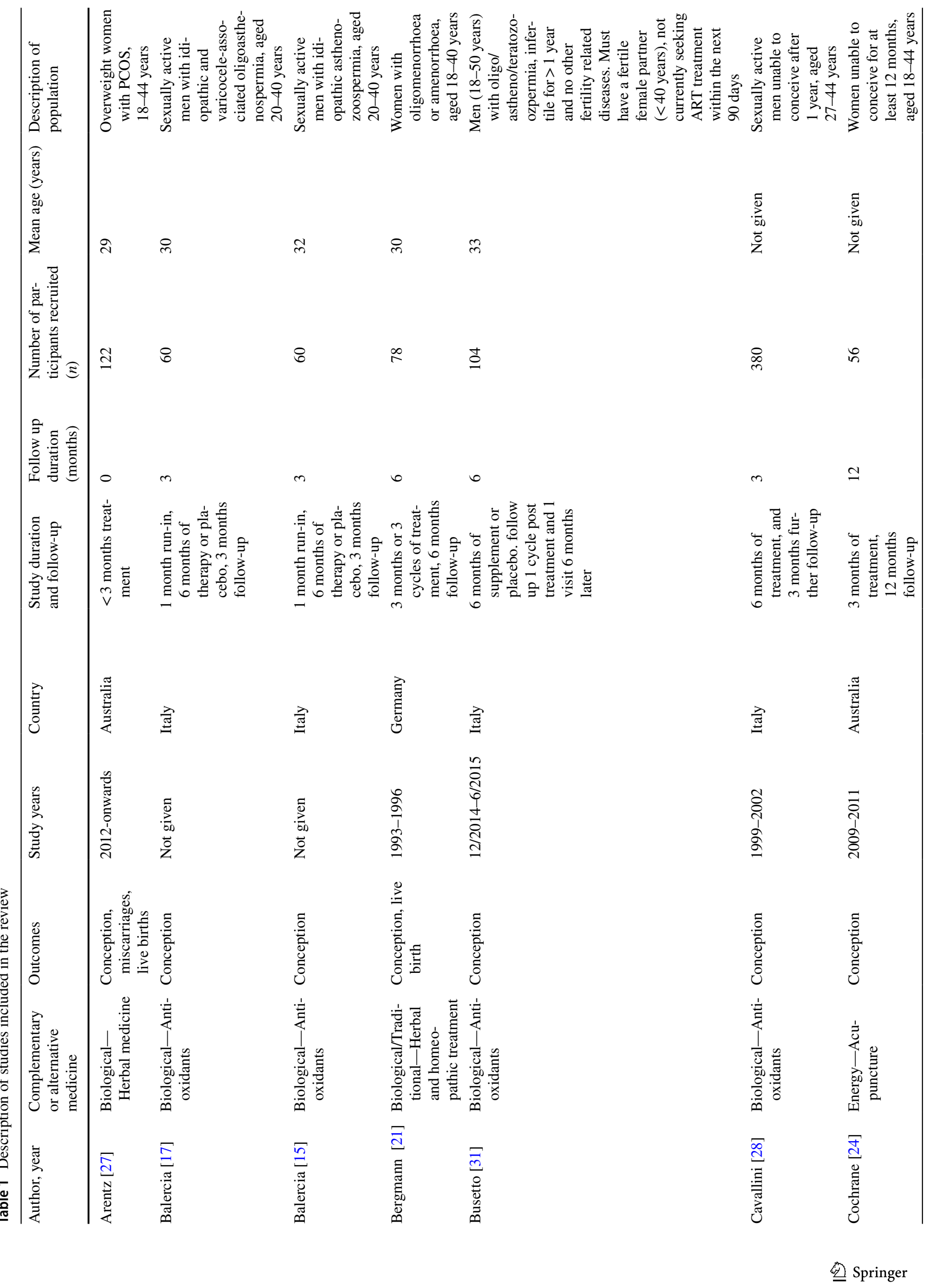




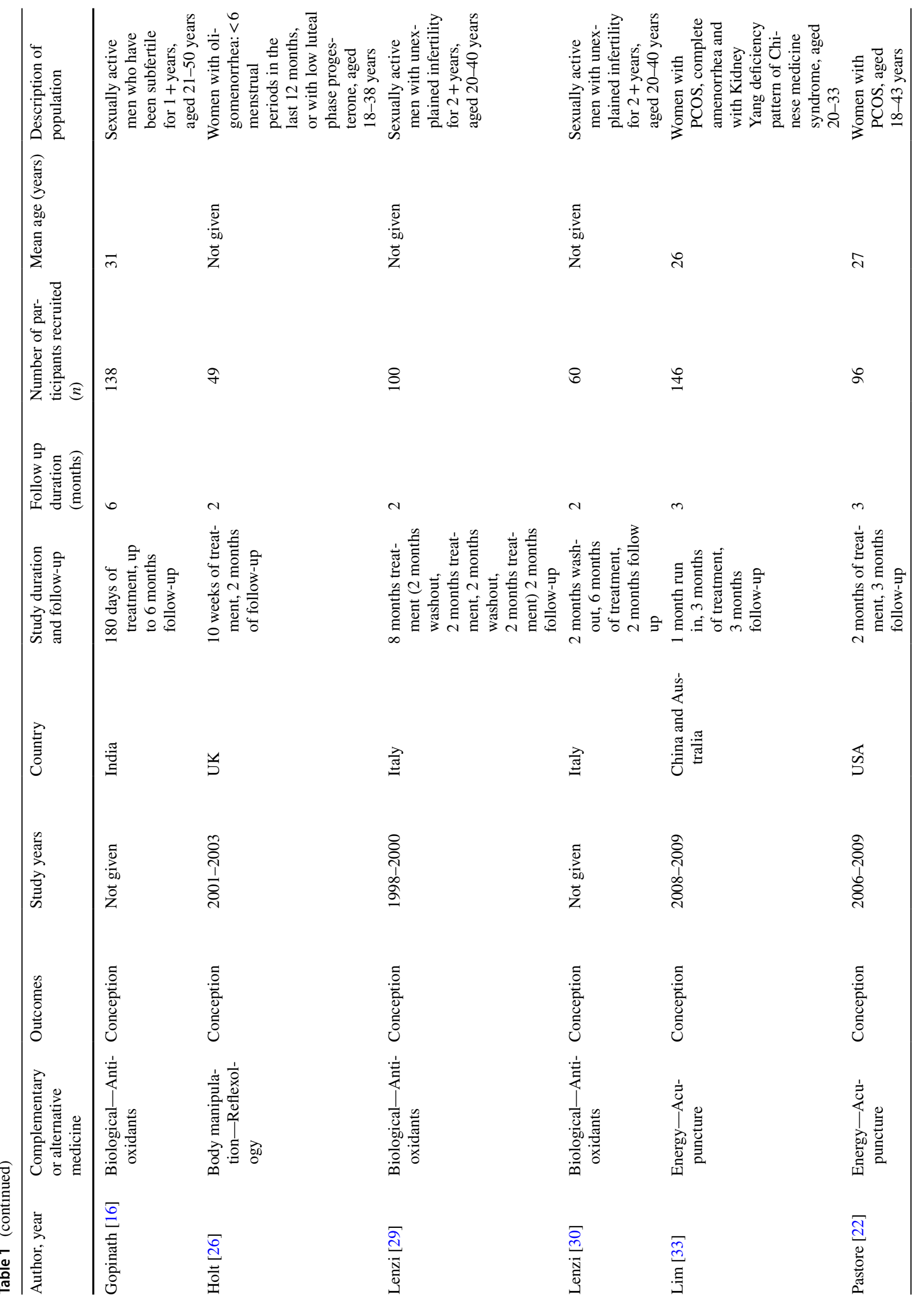




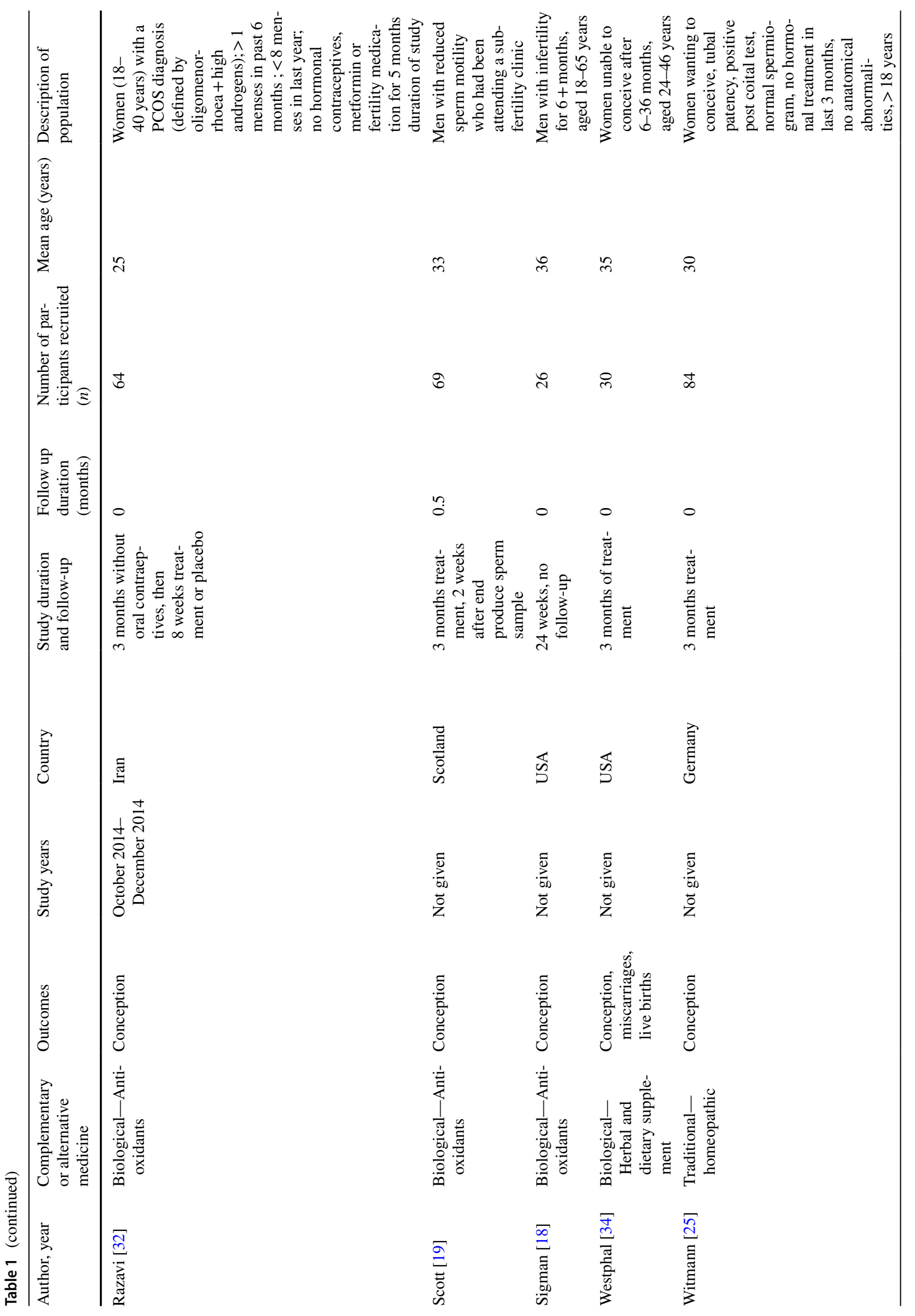




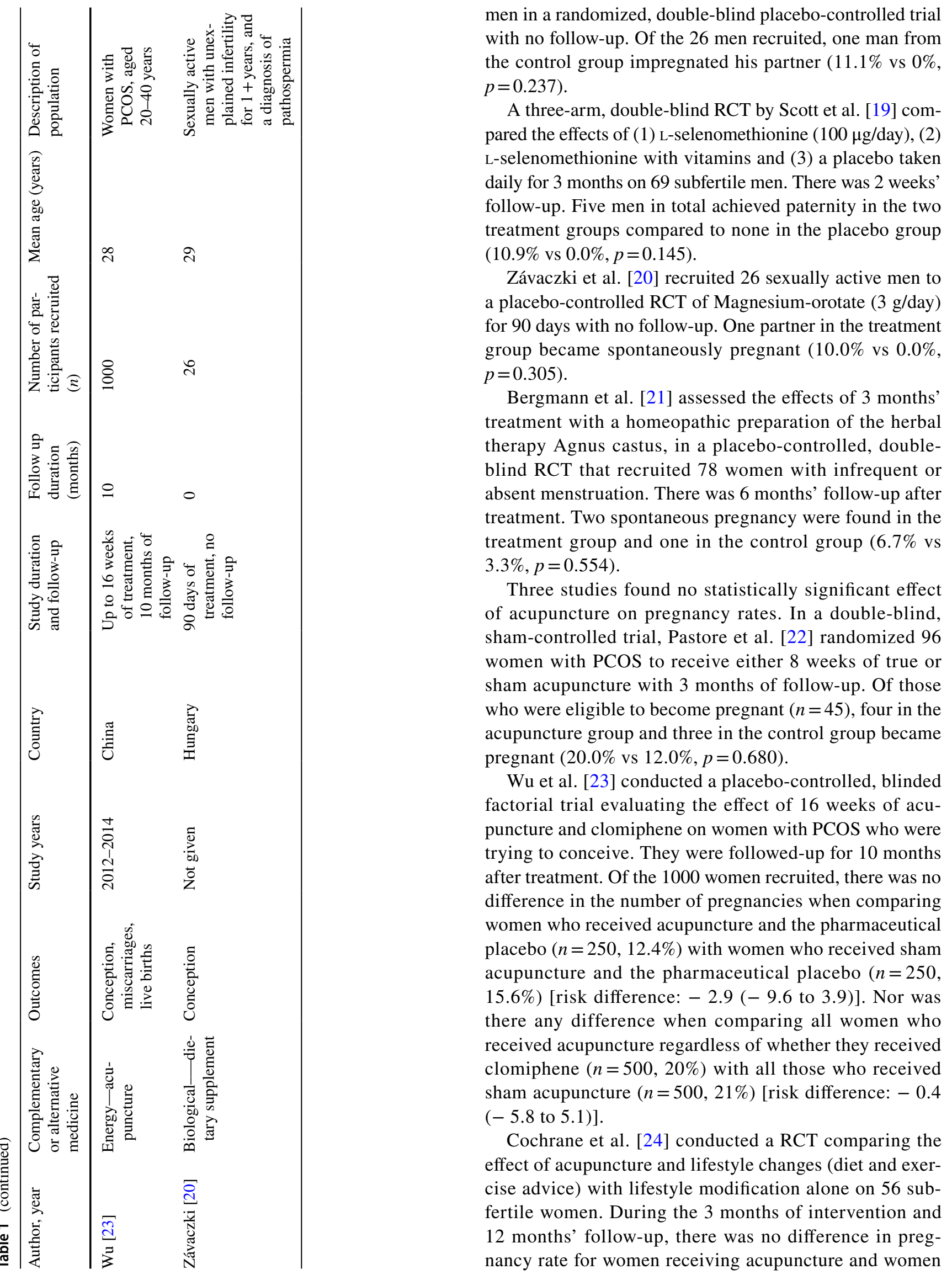



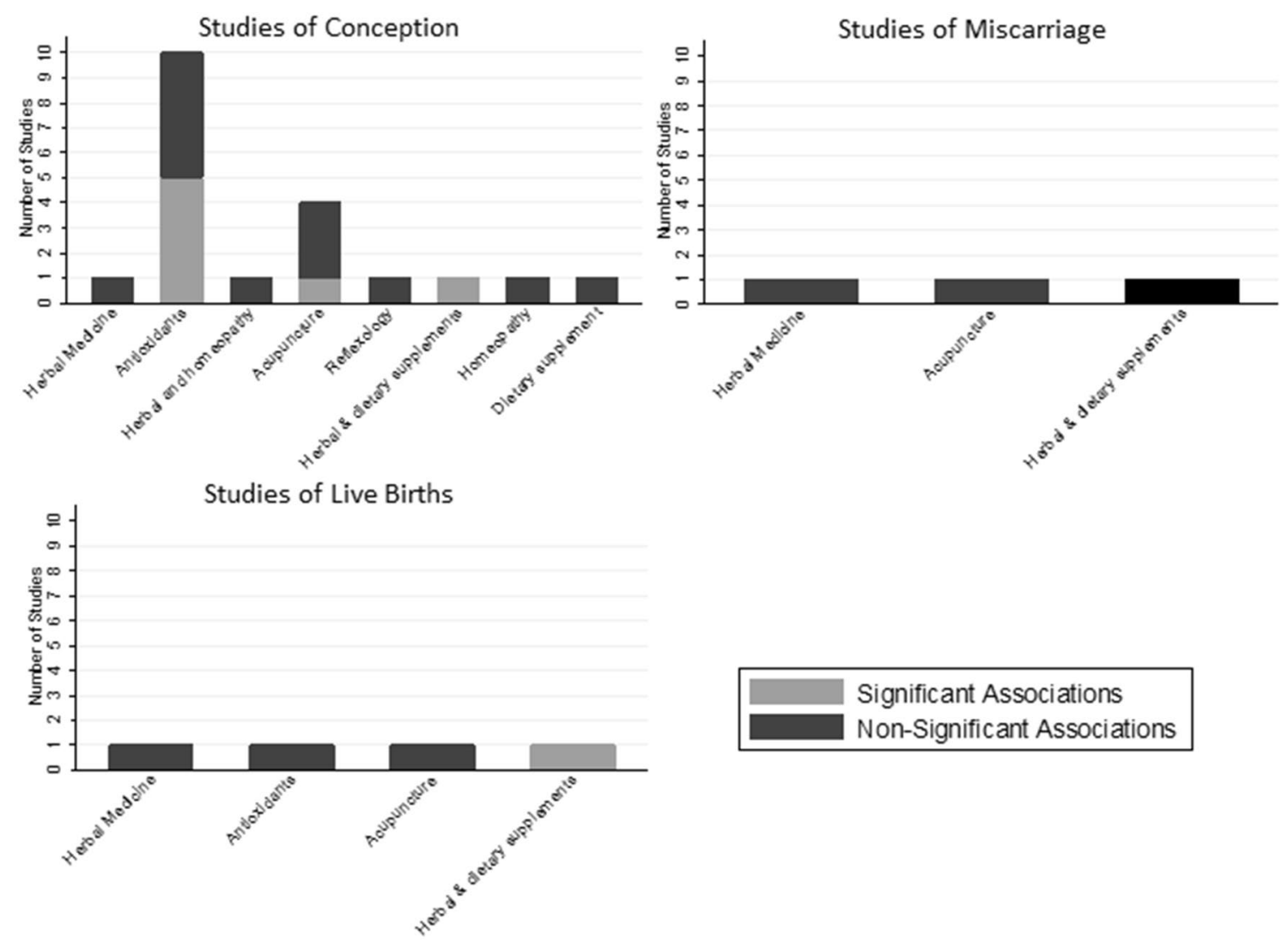

Fig. 2 Summary of the included studies results

Table 2 Quality of evidence evaluating complementary and alternative medicine use and reproductive outcomes

\begin{tabular}{ll}
\hline Effect & Quality of evidence \\
\hline Antioxidants for men do not affect rates of conception $^{\mathrm{a}}$ & Very low (D) \\
Herbal therapies for women improve rates of conception $^{\mathrm{b}}$ & Very low (D) \\
Acupuncture for women does not affect rates of conception $^{\mathrm{c}}$ & Low (C) \\
Homeopathy for women has no statistically significant effect on live birth rates $^{\mathrm{d}}$ & Low (C)
\end{tabular}

GRADE rating was applied when two or more studies assessed the same exposure and outcome. GRADE rating $-\mathrm{C}$ : low, the true effect might be markedly different from the estimated effect; D: very low, the true effect is probably markedly different from the estimated effect

${ }^{a}$ Based on ten studies

${ }^{\mathrm{b}}$ Based on three studies;

${ }^{\mathrm{c}}$ Based on four studies;

${ }^{\mathrm{d}}$ Based on two studies

receiving lifestyle intervention alone $(14.3 \%$ vs $10.7 \%$, $p=0.992$ ). Twelve months following intervention, ten women receiving acupuncture and eight women receiving lifestyle intervention alone had become pregnant in total (35.7\% vs $17.9 \%, p=0.131)$.

The effect of homeopathic therapy on conception was assessed in a double-blinded RCT by Wittmann et al. [25]. 84 women were given 60 drops of Mastodynon or placebo to take daily for 3 months with no additional follow-up.
Nine women each in the treatment and control groups became pregnant $(21.4 \%$ vs $23.1 \%, p=0.494)$.

Finally, Holt et al. [26] conducted a sham-controlled double-blinded RCT of eight sessions of reflexology on 49 women with anovulation over 10 weeks with 2 months of follow-up. Four women in the treatment group and two women in the control group conceived $(19.0 \%$ vs $11.1 \%$, $p=0.680)$. 


\section{Studies reporting an effect}

Arentz et al. [27] assessed the effect of up to 3 months' use of two herbal supplements in 122 overweight women with PCOS receiving diet and exercise advice. There was no follow-up. The treatment group received a tablet combining extracts of Glycyrrhiza glabra, Paeonia lactiflora, Cinnamomum verum and Hypericum perforatum and another containing Tribulus terrestris extract, the latter to be taken during follicular phase of the menstrual cycle. Eleven women in the treatment group became pregnant and three in the control group (32.4\% vs $8.3 \%, p=0.012$ ).

In a double-blind placebo-controlled trial, Westphal et al. [34] investigated the effect of 3 months of FertilityBlend ${ }^{\mathrm{TM}}$, a nutritional supplement that included herbal extracts, antioxidants, vitamins and minerals, on 30 women who had been trying to conceive for 6-36 months. No follow-up period was reported. Five women, all of whom had received the supplement, became pregnant ( $33.3 \%$ vs $0.0 \%, p=0.014)$.

Four trials found evidence for a positive effect of the antioxidants L-carnitine and L-acetyl-carnitine for men with subfertility. Cavallini et al. [28] randomized 325 men to one of three arms. Treatment group 1 received L-carnitine $(2 \mathrm{~g} /$ day) and L-acetyl-carnitine ( $1 \mathrm{~g} /$ day $)$ with a cinnoxicam suppository $(30 \mathrm{mg}$ ) every 4 days. Treatment group 2 received the same doses of L-carnitine and L-acetyl-carnitine and a placebo suppository. The control group received oral and suppository placebos. Treatment was for 6 months with 3 months' further follow-up. Conception rates were significantly higher in treatment group 1 than the control group $(21.8 \%$ vs $1.7 \%, p<0.001)$ and treatment group 2 than the control group (38.0\% vs $1.7 \%, p<0.001)$. Conception rates in treatment group 2 were also significantly higher than in treatment group $1(38.0 \%$ vs $21.8 \%, p=0.012)$.

In two separate placebo-controlled, double-blind, crossover trials Lenzi et al. [29, 30] assessed the effect of antioxidants on sexually active men with sub-fertility. In both trials, treatment was received for 2 months with 2 months' washout period in between treatments and 2 months' followup afterwards. The first trial recruited 100 men and evaluated L-carnitine ( $2 \mathrm{~g} /$ day $)$. No pregnancies occurred during the control periods, but eight pregnancies occurred during treatment $(0.0 \%$ vs $9.3 \%, p=0.004)$. In a separate trial, 60 men alternated receiving L-carnitine ( $2 \mathrm{~g} /$ day) with L-acetylcarnitine $(1 \mathrm{~g} /$ day $)$ or placebo. Four pregnancies occurred during treatment phases and none during placebo phases ( $13.3 \%$ vs $0.0 \%, p=0.045)$.

Busetto et al. [31] investigated the effect of 6 months of antioxidants combined with supplements $(1000 \mathrm{mg} \mathrm{L}$-carnitine, $725 \mathrm{mg}$ fumarate, $500 \mathrm{mg}$ acetyl-L-carnitine, $20 \mathrm{mg}$ co-enzyme Q10, $90 \mathrm{mg}$ vitamin C, $10 \mathrm{mg}$ zinc, $100 \mu \mathrm{g}$ folic acid, $1.5 \mu \mathrm{g} \mathrm{B} 12)$ on 104 subfertile men in a double-blind placebo-controlled RCT. Ten partners in the therapy group became pregnant compared to two in the control group in up to 6 months' follow-up ( $20.4 \%$ vs $4.4 \%, p=0.021)$.

Razavi et al. [32] evaluated the effect of selenium ( $200 \mu \mathrm{g} /$ day) taken for 2 months on 64 women with PCOS in a placebo-controlled RCT with no follow-up. All women received the same dose of metformin over the trial period. Six women taking selenium and one woman taking the placebo became pregnant $(18.8 \%$ vs $3.1 \%, p=0.040)$.

In a RCT by Lim et al. [33] 146 women with PCOS were randomized to 3 months of true or sham acupuncture with 3 months' follow-up. Five women who had received true acupuncture became pregnant whereas none of the women in the control group became pregnant $(5.1 \%$ vs $0.0 \%$, $p=0.002$ ).

\section{Miscarriages}

Three RCTs from Australia, China and USA evaluated the number of miscarriages in men and women when treated with a CAM (eTable 6). The studies evaluated a biological based therapy (a supplement), a body manipulation practice (acupuncture) and a traditional CAM (homeopathy). No studies demonstrated that treatment with a CAM affected rates of miscarriage.

\section{Studies reporting no statistically significant effect}

Arentz et al. [27] evaluated the use of herbal supplements for up to 3 months in women with PCOS with no follow-up. Of the 11 pregnancies that occurred in the treatment group, four ended in miscarriage, while one out of the three pregnancies arising in the control group ended in miscarriage (36.4\% vs $33.3 \%, p=0.923)$.

No statistically significant effect of 16 weeks of acupuncture and 10 months of follow-up on miscarriages for women with PCOS was reported by Wu et al. [23]. The proportion of pregnant women who miscarried was similar in the group receiving acupuncture and a placebo (37.3\%) and the control group $(29.1 \%)$. Compared to the control group, the risk difference of miscarriage for women who had acupuncture and placebo was 8.2 (- 9.7 to 26.1$)$.

Westphal et al. [34] reported one of the five pregnancies that arose in the group receiving 3 months without followup of a herbal and dietary supplement ended in miscarriage (20\%). No pregnancies occurred in the control group and, therefore, no miscarriages.

\section{Live births}

Four RCTs investigated treatment with a CAM on the rates of live births in women (eTable 7). One study each was from Germany, China, Australia and USA. They assessed the effects of a biological based therapy (a supplement), a body 
manipulation practice (acupuncture) and a traditional CAM (homeopathy). Only one study found evidence of greater live birth rates for women treated with a CAM.

\section{Studies reporting no statistically significant effect}

Bergmann et al. [21] conducted a placebo-controlled, double-blind RCT of 3 months of homeopathic preparation of Agnus castus extract, with 3 months' follow-up, recruiting 78 women with infrequent or absent menstruation. There were six live births in the treatment group and two in the control group $(20.0 \%$ vs $6.7 \%, p=0.129)$.

$\mathrm{Wu}$ et al. [23] provided no evidence of an effect of 16 weeks of acupuncture on live birth rates in a placebocontrolled, blinded, factorial trial of clomiphene and acupuncture in women with PCOS that had 10 months' followup. There was no significant difference in the rate of live births between the group receiving active acupuncture and a placebo tablet and the control group (16.8\%) (13.9\%; risk difference, $-2.9 \%$ [95\% CI: $-9.5-3.7 \%)]$.

Arentz et al. [27] provided no evidence of an effect of up to 3 months of homeopathic preparation (containing Glycyrrhiza glabra, Peonia lactiflora, Cinnamonum verum, Hypericum perforatum and Tribulus terrestris) on live birth rates. There was no follow-up. There were seven live births in the treatment group and two in the control group $(20.6 \%$ vs $5.6 \%, p=0.060$ ).

\section{Studies reporting an effect}

A single study found evidence of a higher rate of live births for women receiving a CAM. Westphal et al. [34] randomized women who had tried unsuccessfully to conceive for 6-36 months to receiving 3 months of a herbal and dietary supplement, or a placebo. Although the follow-up duration was not reported, all four live births occurred in the treatment group, resulting from five pregnancies that occurred during and in the year after the trial.

\section{Study quality}

Two out of the 20 included trials demonstrated a high risk of bias, as evaluated using the Cochrane Collaboration tool (Fig. 3, eTable 8). In both trials the high risk of bias related to blinding of participants and personnel. A further 13 trials demonstrated an unclear risk of bias in one or more areas, while 5 trials had a low risk of bias in all areas of study quality.

The GRADE approach was used to determine the quality of evidence for exposure-outcome combinations where two or more studies were identified. The grading based on the current review is indicated in Table 2 and Fig. 4.
Down-grading due to risk of bias, inconsistency and imprecision resulted in low to very low evidence.

\section{Discussion}

\section{Summary of the findings}

This review, based on data from 20 studies, gives an important overview of the current knowledge on effects of CAM for couples not undergoing ART treatments, related to conception, miscarriage and live births. The majority of studies demonstrated no statistically significant effect of CAM on any of the outcomes, and only eight studies reported an advantageous association. Overall, there was low to very low evidence of any CAM effecting any reproductive outcomes.

\section{Findings in context of other research into CAM}

Although women may avoid conventional and pharmacological therapy during pregnancy due to potential adverse effects [35, 36], CAM are often perceived as natural and safe [37]. However, research is lacking on the efficacy and safety of CAM among pregnant and postpartum women even for commonly used herbal therapies [38]. The most pressing issue from couples' perspectives is whether the use of a CAM will affect their risk of pregnancy loss or having a live born child. Only 3 out of the 20 identified studies reported the number of miscarriages, and only 4 studies evaluated the probability of having a live born child. Furthermore, no studies evaluated time to pregnancy. This marked lack of research in this area will only compound the limited advice and guidance that caregivers can provide to couples who wish to have a child.

The focus on this review, on CAM use outside of ART treatment, does not negate potential benefits of CAM use for couples seeking to conceive with ART treatment or for women during pregnancy. A previous systematic review of the evidence from trials for CAM during infertility treatment found preliminary evidence of the effectiveness of some CAM interventions [9]. Three or more clinical trials reported results that were consistent with a positive effect of the use of acupuncture, selenium supplementation, weight loss and psychotherapy during infertility treatment. Acupuncture has also been reported to be beneficial as an adjunct to standard analgesia during oocyte retrieval for IVF [9] and to have a beneficial effect on back pain during pregnancy [23]. 


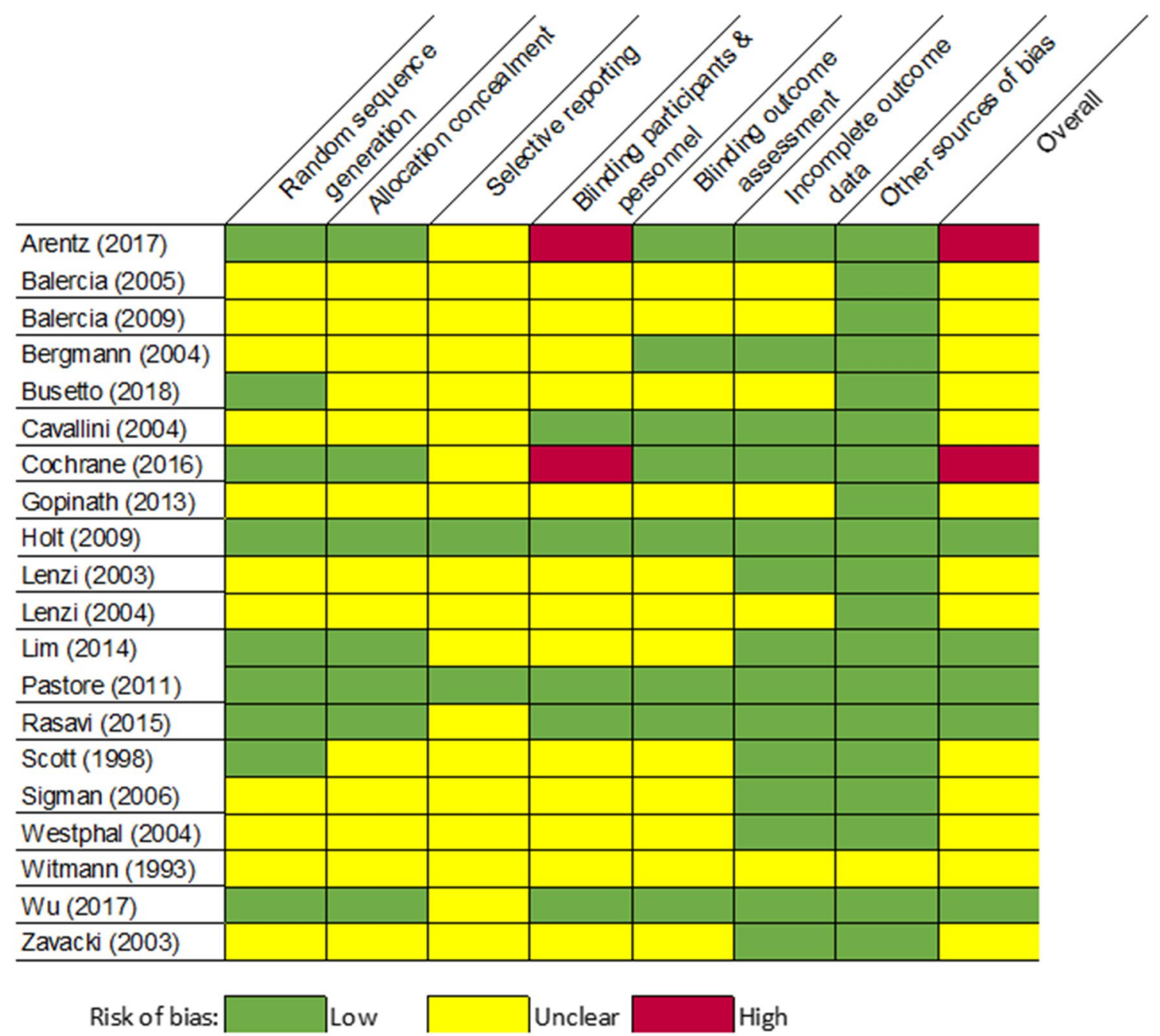

Fig. 3 Quality of studies in the review as assessed by the Cochrane Collaboration's tool

\section{Strengths and limitations of the current evidence and directions for future research}

To the authors' knowledge this is the first review of CAM use in couples not undergoing ART treatment. Strengths include the wide range of CAM included in the search terms and multiple databases used in the search, although this limited detailed evaluation of each CAM. Synthesis of the existing knowledge on this topic was challenging due to inconsistent findings between some studies evaluating similar CAM (e.g. Balercia et al. [17] and Cavallini et al. [28]), which may partly reflect differences in the dose, formulation or route of administration of CAM used in studies theoretically evaluating the same exposure. Publication bias may also have impacted the review, although the majority of the studies have small sample sizes and did not report an effect on reproductive outcomes. Furthermore, the majority of included studies are from European populations, which might limit the generalizability of the findings to other populations, and the quality of studies was generally poor. Other important factors such as differences in underlying fertility risk factors in study populations and variability in adjustment levels prevent quantitative synthesis of the studies.

The importance of age in the included studies cannot be overstated. A major determinant of fertility in women, age impacts ovarian reserves and, therefore, the quantity and quality of oocytes available for fertilization. The ability to become pregnant at the age of 34-35 is $14 \%$ lower than at the ages of $30-31$, which decreases to $50 \%$ by the age of 40 [39]. Therefore, the variation in the mean age of female participants in the included studies, from 26 to 34, will have a large effect on the probability of conception and introduced between-study heterogeneity to this review.

Our review should stimulate further research in this area. The aforementioned few studies assessing miscarriage, live birth rates and time to pregnancy is a major gap in the 
Fig. 4 Clinical implications of findings on complementary and alternative medicines and reproductive outcomes. Quality of evidence assessed using Grading of Recommendations Assessment, Development and Evaluation (GRADE): C: low quality of evidence (our confidence in the effect estimate is limited: The true effect may be substantially different from the estimate of the effect). D: very low quality of evidence (out confidence in the effect estimates is severely limited. The true effect is probably markedly different from the estimated effect)

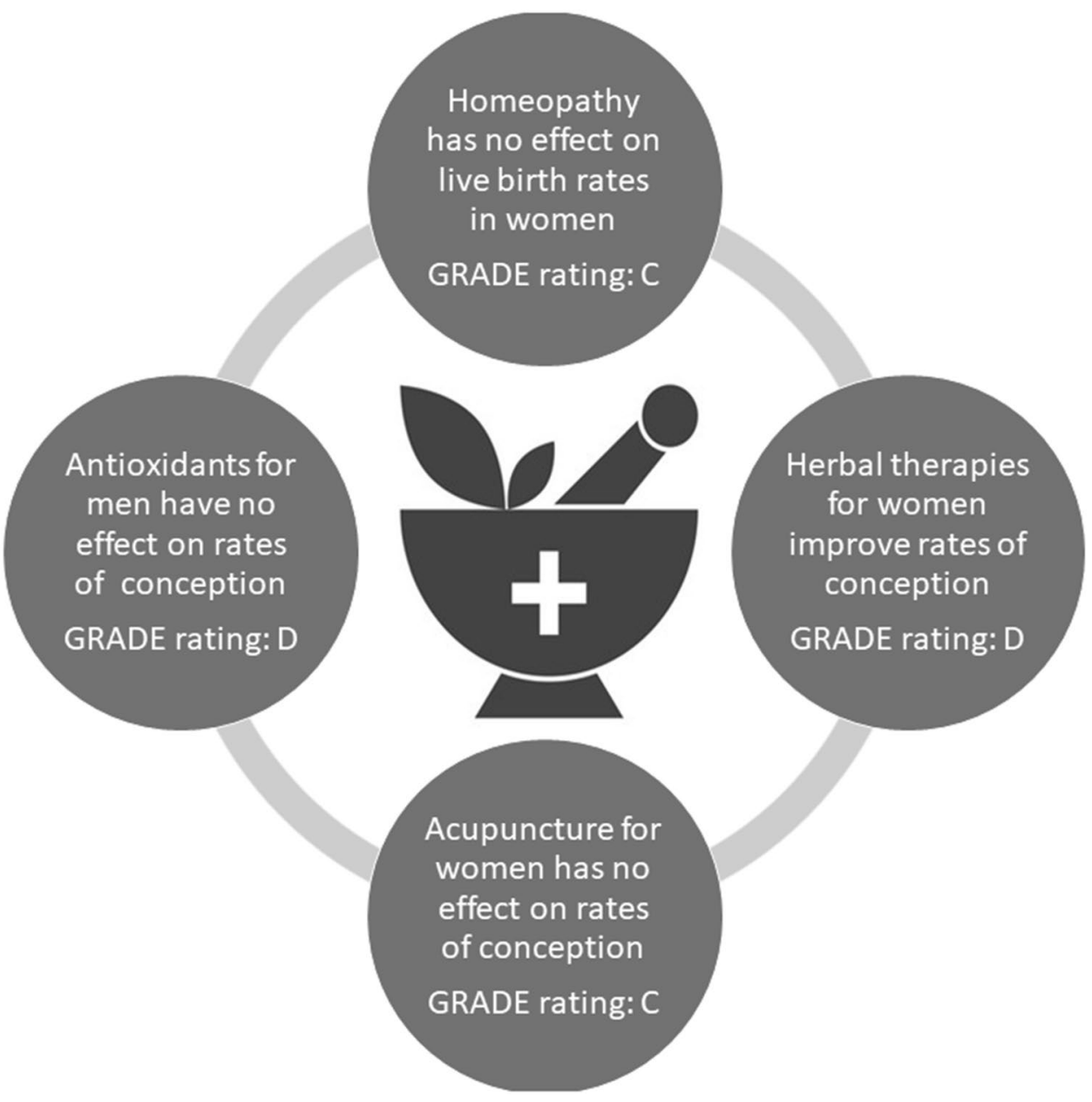

literature. The latter is particularly pertinent; many studies assessed conceptions but neglected to conduct any statistical analyses assessing time to pregnancy. Furthermore, the majority of identified studies assessed biological CAM. In general, the small number of individuals included in most of the studies (number of participants recruited: mean $=137$, median $=74$ ) strongly suggests the studies may lack power to detect any significant effect of a CAM and call into question whether significant results reflect chance findings.

To comprehensively investigate the role of CAM, it may be necessary to conduct large population-based studies rather than RCTs. Previous surveys indicate that large numbers of couples use CAM when trying to get pregnant [8] or during pregnancy [40]. Given the small numbers of participants in the trials included in this review (18 studies had $<200$ participants), and the relatively short follow-up durations (range: 0-12 months), a cohort study with large numbers of participants followed for a sufficient amount of time ( $>5$ years) may be more feasible. This would also have the advantage of including women and men with a wide range of ages and, therefore, having the potential to evaluate the effects of a CAM at different ages. A good example of this is the cohort study of over 25,000 individuals evaluating whether use of acupuncture after stroke reduced the risk of pneumonia [41].

\section{Conclusion}

The current review presents a cutting-edge summary of CAM and pregnancy outcomes. However, the findings of this article should be treated cautiously. The quality of many studies included in this review was low, although findings were based on RCTs. Use of CAM should be based on evidence and attention should be paid to negative effects, such as a potential increased risk of miscarriage. Also, it is recommended that medical professionals discuss with patients who approach them with fertility problems about whether they are using any CAM. Although no negative effects on conception were reported, side effects were prevalent. These include diarrhea [20], euphoria [28] and headaches [24].

Overall, the evidence on CAM and pregnancy outcomes for individuals not using ART treatment is not robust due to low study quality and small sample sizes. There is limited 
support for the role of different CAM, such as herbal therapies in enhancing the chances of conception and subsequent live births.

Author contributions CO-W: protocol/project development, data collection or management, data analysis, manuscript writing/editing. SQ: protocol/project development, manuscript writing/editing. EB: proto$\mathrm{col} /$ project development, data collection or management, manuscript writing/editing. CYSS, AJOH, HMY, WJW, ACC: data collection or management, manuscript writing/editing.

Funding This research was not supported by any grant. CO holds a fellowship at Homerton College, University of Cambridge. The Department of Public Health and Primary Care received funding from the British Heart Foundation and the Medical Research Council, UK. None of the sources of funding had any involvement in study design; data collection, analysis and interpretation of data; writing of the report or decision to submit the report for publication.

Availability of data and materials All data generated or analyzed during this study are included in this published article and its supplementary information files.

\section{Compliance with ethical standards}

Conflict of interest Dr Quenby reports grants from Tommy's baby Charity, during the conduct of the study. CO, EB, AH, CS, WW, ACC, HMY declare that they have no competing interests.

Open Access This article is licensed under a Creative Commons Attribution 4.0 International License, which permits use, sharing, adaptation, distribution and reproduction in any medium or format, as long as you give appropriate credit to the original author(s) and the source, provide a link to the Creative Commons licence, and indicate if changes were made. The images or other third party material in this article are included in the article's Creative Commons licence, unless indicated otherwise in a credit line to the material. If material is not included in the article's Creative Commons licence and your intended use is not permitted by statutory regulation or exceeds the permitted use, you will need to obtain permission directly from the copyright holder. To view a copy of this licence, visit http://creativecommons.org/licenses/by/4.0/.

\section{References}

1. Oakley L, Doyle P, Maconochie N (2008) Lifetime prevalence of infertility and infertility treatment in the UK: results from a population-based survey of reproduction. Hum Reprod $23: 447-450$

2. Stephen EH, Chandra A (2006) Declining estimates of infertility in the United States: 1982-2002. Fertil Steril 86:516-523

3. Harris K, Burley H, McLachlan R, Bowman M, Macaldowie A, Taylor K et al (2016) Socio-economic disparities in access to assisted reproductive technologies in Australia. Reprod Biomed Online 33:575-584

4. Huddleston HG, Cedars MI, Sohn SH, Giudice LC, Fujimoto VY (2010) Racial and ethnic disparities in reproductive endocrinology and infertility. Am J Obstet Gynecol 202:413-419
5. Quinn M, Fujimoto V (2016) Racial and ethnic disparities in assisted reproductive technology access and outcomes. Fertil Steril 105:1119-1123

6. Adashi EY, Dean LA (2016) Access to and use of infertility services in the United States: framing the challenges. Fertil Steril 105:1113-1118

7. Vincent C, Furnham A (1996) Why do patients turn to complementary medicine? An empirical study. Br J Clin Psychol 35:37-48

8. Smith JF, Eisenberg ML, Millstein SG, Nachtigall RD, Shindel AW, Wing $\mathrm{H}$ et al (2010) The use of complementary and alternative fertility treatment in couples seeking fertility care: data from a prospective cohort in the United States. Fertil Steril 93:2169-2174

9. Clark NA, Will M, Moravek MB, Fisseha S (2013) A systematic review of the evidence for complementary and alternative medicine in infertility. Int J Gynecol Obstet 122:202-206

10. National Institute for Health Research (2018) PROSPERO: International prospective register of systematic reviews. https://www. crd.york.ac.uk/prospero/. Accessed 19 Mar 2020

11. Allaire AD, Moos MK, Wells SR (2000) Complementary and alternative medicine in pregnancy: a survey of North Carolina certified nurse-midwives. Obstet Gynecol 95:19-23

12. Miner SA, Robins S, Zhu YJ, Keeren K, Gu V, Read SC et al (2018) Evidence for the use of complementary and alternative medicines during fertility treatment: a scoping review. BMC Complement Altern Med 18:158

13. Strouss L, Mackley A, Guillen U, Paul DA, Locke R (2014) Complementary and alternative medicine use in women during pregnancy: Do their healthcare providers know? BMC Complement Altern Med 14:85

14. Higgins JPT, Altman DG, Gøtzsche PC, Jüni P, Moher D, Oxman $\mathrm{AD}$ et al (2011) The cochrane collaboration's tool for assessing risk of bias in randomised trials. BMJ 343:d5928

15. Balercia G, Buldreghini E, Vignini A, Tiano L, Paggi F, Amoroso $S$ et al (2009) Coenzyme Q10 treatment in infertile men with idiopathic asthenozoospermia: a placebo-controlled, double-blind randomized trial. Fertil Steril 91:1785-1792

16. Gopinath PM, Kalra B, Saxena A, Malik S, Kochhar K, Kalra $S$ et al (2013) Fixed dose combination therapy of antioxidants in treatment of idiopathic oligoasthenozoospermia: results of a randomized, double-blind, placebo-controlled clinical trial. Int J Infertil Fetal Med 4:6-13

17. Balercia G, Regoli F, Armeni T, Koverech A, Mantero F, Boscaro M (2005) Placebo-controlled double-blind randomized trial on the use of L-carnitine, L-acetylcarnitine, or combined L-carnitine and L-acetylcarnitine in men with idiopathic asthenozoospermia. Fertil Steril 84:662-671

18. Sigman M, Glass S, Campagnone J, Pryor JL (2006) Carnitine for the treatment of idiopathic asthenospermia: a randomized, doubleblind, placebo-controlled trial. Fertil Steril 85:1409-1414

19. Scott R, Macpherson A, Yatest RWS, Hussain B, Dixon J (1998) The effect of oral selenium supplementation on human sperm motility. Br J Urol 82:76-80

20. Závaczki Z, Szöllõsi J, Kiss SA, Koloszár S, Fejes I, Kovács L et al (2003) Magnesium-orotate supplementation for idiopathic infertile male patients: a randomized, placebo-controlled clinical pilot study. Magnes Res. 16:131-136

21. Bergmann J, Luft B, Boehmann S, Runnebaum B, Gerhard I (2000) Die Wirksamkeit des Komplexmittels Phyto-Hypophyson ${ }^{\circledR} \mathrm{L}$ bei weiblicher, hormonell bedingter Sterilität. Eine randomisierte, placebokontrollierte, klinische Doppelblindstudie. Complement Med Res. 7:190-199

22. Pastore LM, Williams CD, Jenkins J, Patrie JT (2011) True and sham acupuncture produced similar frequency of ovulation and improved LH to FSH ratios in women with polycystic ovary syndrome. J Clin Endocrinol Metab 96:3143-3150 
23. Wu XK, Stener-Victorin E, Kuang HY, Ma HL, Gao JS, Xie LZ et al (2017) Effect of acupuncture and clomiphene in Chinese women with polycystic ovary syndrome: a randomized clinical trial. JAMA J Am Med Assoc 317:2502-2514

24. Cochrane S, Smith CA, Possamai-Inesedy A, Bensoussan A (2016) Prior to conception: the role of an acupuncture protocol in improving women's reproductive functioning assessed by a pilot pragmatic randomised controlled trial. Evid Based Complement Alternat Med 2016:3587569

25. Wittmann G, Gerhard I, Runnebaum B (1993) Wirksamkeit eines Arzneimittels auf Basis pfanzlicher Extrakte Mastodynon-NR bei weiblicher Infertilität. Arch Gynecol Obstet 254:158-160

26. Holt J, Lord J, Acharya U, White A, O'Neill N, Shaw S et al (2009) The effectiveness of foot reflexology in inducing ovulation: a sham-controlled randomized trial. Fertil Steril 91:2514-2519

27. Arentz S, Smith CA, Abbott J, Fahey P, Cheema BS, Bensoussan A (2017) Combined lifestyle and herbal medicine in overweight women with polycystic ovary syndrome (PCOS): a randomized controlled trial. Phyther Res 31:1330-1340

28. Cavallini G, Ferraretti AP, Gianaroli L, Biagiotti G, Vitali G (2004) Cinnoxicam and L-carnitine/acetyl-L-carnitine treatment for idiopathic and varicocele-associated oligoasthenospermia. J Androl 25:761-770

29. Lenzi A, Lombardo F, Sgrò P, Salacone P, Caponecchia L, Dondero F et al (2003) Use of carnitine therapy in selected cases of male factor infertility: a double-blind crossover trial. Fertil Steril 79:292-300

30. Lenzi A, Sgrò $\mathrm{P}$, Salacone $\mathrm{P}$, Paoli D, Gilio B, Lombardo F et al (2004) A placebo-controlled double-blind randomized trial of the use of combined L-carnitine and L-acetyl-carnitine treatment in men with asthenozoospermia. Fertil Steril 81:1578-1584

31. Busetto GM, Agarwal A, Virmani A, Antonini G, Ragonesi G, Del Giudice F et al (2018) Effect of metabolic and antioxidant supplementation on sperm parameters in oligo-astheno-teratozoospermia, with and without varicocele: a double-blind placebocontrolled study. Andrologia. 50:e12927

32. Razavi M, Jamilian M, Fakhrieh Kashan Z, Heidar Z, Mohseni M, Ghandi Y et al (2016) Selenium supplementation and the effects on reproductive outcomes, biomarkers of inflammation, and oxidative stress in women with polycystic ovary syndrome. Horm Metab Res 48:185-190

33. Lim CED, Luo X, Zhan X, Wong WSF (2014) ACUPCOS: acupuncture \& PCOS - a multi-centres randomised controlled trial. Int J Gynecol Obstetr Res 2:1-13

34. Westphal LM, Polan ML, Trant AS, Mooney SB (2004) A nutritional supplement for improving fertility in women: a pilot study. J Reprod Med Obstet Gynecol 49:289-293

35. Nordeng H, Koren G, Einarson A (2010) Pregnant women's beliefs about medications-a study among 866 Norwegian women. Ann Pharmacother 44:1478-1484

36. McDonald K, Amir LH, Davey M-A (2011) Maternal bodies and medicines: a commentary on risk and decision-making of pregnant and breastfeeding women and health professionals. BMC Public Health 11(Suppl 5):S5

37. Adams J, Lui C-W, Sibbritt D, Broom A, Wardle J, Homer C et al (2009) Women's use of complementary and alternative medicine during pregnancy: a critical review of the literature. Birth $36: 237-245$

38. Holst L, Wright D, Haavik S, Nordeng H (2011) Safety and efficacy of herbal remedies in obstetrics-review and clinical implications. Midwifery 27:80-86

39. Steiner AZ, Jukic AMZ (2016) Impact of female age and nulligravidity on fecundity in an older reproductive age cohort. Fertil Steril 105(1584-1588):e1

40. Birdee GS, Kemper KJ, Rothman R, Gardiner P (2014) Use of complementary and alternative medicine during pregnancy and the postpartum period: an analysis of the national health interview survey. J Women's Heal 23:824-829

41. Chang C-C, Chen T-L, Lin C-S, Chung C-L, Yeh C-C, Hu C-J et al (2018) Decreased risk of pneumonia in stroke patients receiving acupuncture: A nationwide matched-pair retrospective cohort study. PLoS ONE 13:e196094

Publisher's Note Springer Nature remains neutral with regard to jurisdictional claims in published maps and institutional affiliations. 